\title{
Research on seat perceived comfort evaluation based on factor analysis
}

\author{
Yu Qiao*, Changjiang Du, Yunpeng Cong, Zeyang Tian, Shuhang Wang, and Zhun Zhao \\ CATARC Automotive Test Cent (Tianjin) Co.,Ltd. No.68, East Xianfeng Road, Dongli District, \\ Tianjin, China
}

\begin{abstract}
Car seat is one of the most important parts of the direct contact with consumers, consumers' subjective perception of seat comfort depends on the choice of car, and the automobile industry in the perception of car seat comfort evaluation and research work, is still in its infancy, convened by the project through to the six major cities, 900 consumers respectively for 18 to subjective comfort evaluation of standard models, this article selects three same level models of standard models, for example, through the factor analysis method to calculate the subjective comfort ratings, and use the objective measurement data of subjective rating transverse comparison test. The results show that the subjective comfort score can reflect the comfort degree of the seat.
\end{abstract}

Keywords: Car Seat; Perceived Comfort; Factor Analysis.

\section{Introduction}

In recent years, with the progress and development of automotive technology, people's requirement about vehicle comfort increased constantly, comfort has become one of the most factors gained consumers' attention. According to the research from CATARC, in 2015 and 2016, comfort was the most primary concern of consumers in all the product indicators. Even in 2017, comfort was concerned secondly, following the quality. From the vehicle riding comfort, space comfort, easy to operate and other aspects of vehicle comfort, as one of the most important parts contact with consumers directly, seat is one of the most important elements to effect vehicle comfort. We can say, seat comfort is the most vital part forming the vehicle comfort. Being one of the most direct feeling to vehicle comfort of consumers, seat perceived comfort became one of the most vital factor while consumers purchasing cars $^{[1]}$.

This article focuses on seat assemblies in different quality (Involve brand, material etc.), implementing the factor analysis about subjective perceived comfort, from the dimensions of smell, touch, vision, hearing and so on.

\footnotetext{
* Corresponding author: qiaoyu@catarc.ac.cn
} 


\section{Source of subjective comfort evaluation data}

This article set up subjective evaluation questionnaire base on the seat perceived comfort evaluation system, setting different types of evaluation questions which focus on the main design area of the seat, (head pillow, shoulder, chest, waist, side of back, coccyx, hip, front of thigh, side of seat cushion) and let the consumers grade one by one. As figure 1 showing, the means to evaluate comfort of different parts on the seat have some differences. For perceived comfort project, both the consumers' evaluations, about sight, hearing, smell and touch, have one-way attribute. This article defined grade level questions to evaluate projects of the seat. Through the work evaluating and verifying the seat perceived comfort that carried out by project team, gathered approximately 600 consumers from 6 big cities all around the country then conducted them to research for a total of 18 standard models which belong to three types, and record the data as main source for subsequent subjective evaluation analysis.
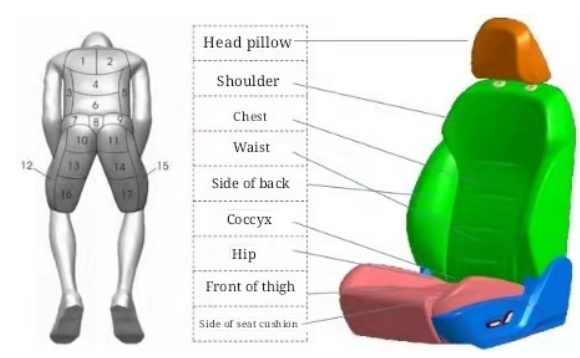

Fig. 1. Different parts of the seat correspond to the human body.

Grade level questions mainly aim to the terms have one-way attribute in comfort evaluation, the score normally set as 1-10, higher score means better comfort. For example, if the front and back adjustment of the seat get the score 1, it means that the noise of the front and back adjustment is very loudly, and the auditory comfort is unacceptable; if the score is 10 , indicating that the seat's front and back adjustment is quiet and the movement sounds very comfortable.

Let consumers from their own feeling about hearing, smell and touch, based on the appearance, structure and function to score, so that achieving comfort evaluation.

By selecting a total of 1,800 people in six domestic cities then evaluated with questionnaires respectively, about three types, including compact SUV, medium SUV and medium car. The scores of relevant sample types under different evaluation dimensions were taken as the original sample data.

\section{Subjective comfort evaluation data processing}

Factor analysis is the decomposition of a set of variables from sample data into a linear combination of a set of potentially dominant common factors and special factors. Simply speaking, the purpose of factor analysis is to reveal the internal relevance between measured variables. Under the precondition of saving original information as much as possible, using fewer dimensions to represent original data structure and to simplify the data, convenient the discovery of laws and essence ${ }^{[2-5]}$. This section takes Honda VEZEL as an example for data processing.

\subsection{Standardized processing and suitability test}

In order to avoid the deviation carried by dimensional difference of samples in calculation 
and analysis process, and also to avoid the interference caused by the difference of data units to overall data analysis, meanwhile for ensuring the objectivity and strictness of the analysis, the statistical data were standardized firstly. After standardized processing, although dimensional is unified, not all data are suitable for data analysis which using factor analysis method. Therefore, the suitability test named KMO test, need to be done for sample. The KMO value is between 0 and 1 , also the KMO value closer to 1 , the variable relevance coefficient higher, the sample is more suitable for factor analysis. While the $\mathrm{KMO}$ value closer to 0 , lower the variable relevance coefficient is, the sample is more unsuitable for factor analysis.

Table 1. KMO and Bartlett tests of vehicle 1 data.

\begin{tabular}{ccc}
\hline \multicolumn{2}{c}{ KMO measure of sampling adequacy } & 0.958 \\
\hline \multirow{2}{*}{$\begin{array}{c}\text { Bartlett test of } \\
\text { sphericity }\end{array}$} & $\begin{array}{c}\text { Approximate } \\
\text { chi-square }\end{array}$ & 25410.668 \\
\cline { 2 - 3 } & degree of freedom & 136 \\
\cline { 2 - 3 } & significance & 0.000 \\
\hline
\end{tabular}

Table 1 shows the result of KMO test and Bartlett test of Car No.1 data. As what is shown by the figure, the KMO value is 0.958 , very close to 1 , this proving that the KMO test has considerable good effect. Bartlett spherical test is to test whether the correlation matrix is the identity matrix. According to the results in Table 1, we can find the adjoint probability value is $0.000<0.001$, and the result meets a significant level. Refusing the null hypothesis, that is, the correlation matrix is not the identity matrix, indicating that there are common factors exist among the relevance matrices of the parent group. The results of both $\mathrm{KMO}$ test and Bartlett test indicate that the data is very suitable for factor analysis.

\subsection{Correlation analysis of data}

The correlation is to study the strength and convergence of the linear correlation between two variables in the sample data by analyzing the sample. In some degree, the possibility of whether the change of one variable will affect the change of another variable, can be reflected by analysis-obtained correlation coefficient.

According to the correlation statistics of the characteristics of subjective comfort evaluation index, the correlation results are also between $0-1$, and the closer the value is to 1 , the better the correlation between them is, while the closer the value is to 0 , the worse the correlation is. Taking visual comfort index as an example, it can be found that in the six visual comfort indexes, the correlation of any two indexes is greater than 0.6 , and the maximum correlation is close to 0.8 , which proves that the correlation between visual comfort indexes is good. Most of the correlation coefficients between visual comfort and auditory comfort, olfactory comfort and tactile comfort are between 0.3 and 0.5 , indicating that the correlation between visual comfort and auditory comfort, olfactory comfort and tactile comfort is poor.

Similarly, the correlation between auditory comfort subjective evaluation indexes is good, but the correlation with other subjective evaluation indexes is poor. The same situation, the correlation between tactile comfort subjective evaluation indexes is good, but the correlation with other subjective evaluation indexes is poor. There is only one index of olfactory comfort, the olfactory comfort has poor correlation with all other comfort evaluation indexes.

It can be concluded through the analysis of the correlation coefficient evaluation results, the correlation is good between the subjective comfort evaluation indexes those in the same category, but the correlation is poor between the subjective comfort evaluation indexes those in different categories. 


\subsection{Extraction factor}

After KMO test, Bartlett test and correlation analysis, further processing the data, named factor load analysis and total variance interpretation.

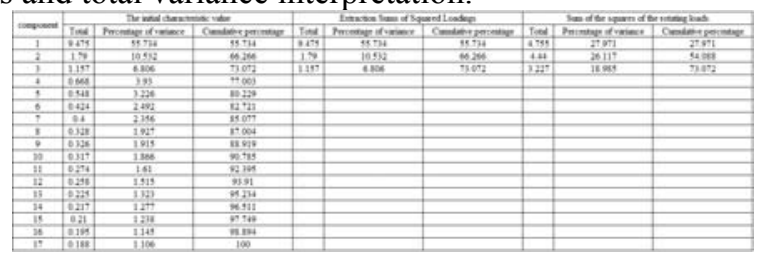

Fig. 2. Total variance interpretation of initial factors

Figure 2 illustrates the total variance figure of data initial factor processing. In SPSS analysis, the standard of extraction we've ruled is eigenvalues must greater than 1 , what can be known from the figure 4 is that the initial characteristic value of component 1, component 2 and component 3 was 9.475, 1.790 and 1.157, respectively, both of them are greater than 1 . Therefore, three components with a characteristic value greater than 1 were extracted, this is equal extracting three common factors. The variance interpretation of the three factors reached $73.072 \%$, which retained more variable information and had better representativeness. The explanatory degree of factor 1 is $55.734 \%$, factor 2 is $10.532 \%$ and factor 3 is $6.806 \%$.

Therefore, extracting a total of three common factors. In order to see the internal relationship between the sample indexes further, the Caesar normalizing maximum variance method was adopted to orthogonal rotation of the factors, so as to obtain the rotated factor loading matrix, as shown in Table 2.

Table 2. Component matrix after rotation

\begin{tabular}{lccc}
\cline { 2 - 4 } \multicolumn{1}{c}{ Comfort factor } & \multicolumn{3}{c}{ component } \\
\cline { 2 - 4 } & 1 & 2 & 3 \\
\hline Auditory comfort 3 & 0.827 & 0.252 & 0.233 \\
Auditory comfort 2 & 0.825 & 0.256 & 0.240 \\
Auditory comfort 4 & 0.820 & 0.234 & 0.268 \\
Auditory comfort 1 & 0.817 & 0.265 & 0.222 \\
Auditory comfort 5 & 0.789 & 0.237 & 0.262 \\
Auditory comfort 7 & 0.738 & 0.234 & 0.264 \\
Olfactory comfort & 0.374 & 0.295 & 0.373 \\
Visual comfort 3 & 0.222 & 0.829 & 0.245 \\
Visual comfort 4 & 0.235 & 0.818 & 0.233 \\
Visual comfort 2 & 0.229 & 0.807 & 0.289 \\
Visual comfort 1 & 0.211 & 0.785 & 0.310 \\
Visual comfort 5 & 0.260 & 0.751 & 0.204 \\
Visual comfort 6 & 0.386 & 0.678 & 0.195 \\
Tactile comfort 3 & 0.289 & 0.256 & 0.815 \\
Tactile comfort 2 & 0.277 & 0.297 & 0.809 \\
Tactile comfort 1 & 0.261 & 0.355 & 0.730 \\
Tactile comfort 4 & 0.314 & 0.240 & 0.701 \\
\hline
\end{tabular}

It can be concluded from the data in Table 2 that the first principal component has a large load on the auditory comfort, so it can be seen that the evaluation index of auditory comfort is mainly interpreted by the first principal component, similarly, the visual comfort evaluation index is mainly explained by the second principal component, the tactile comfort evaluation index is mainly explained by the third principal component.

The first principal component factor can be called as auditory comfort factor, the second as visual comfort factor, and the third as tactile comfort factor. 


\subsection{Weight calculation}

After gaining three principal components of Car No. 1 and component matrix of principal components by analysis and statistics. In order to compare the influence degree of each subjective comfort evaluation index more intuitively, it has need to calculate the weight of each subjective evaluation index further.

Before calculating the proportion weight of the index, the linear combination coefficient is obtained by starting with the variance of the principal component and the characteristic root of each principal component. By formula: normalized number/square root of corresponding principal component characteristic root. The calculation results are as follows:

Table 3. Coefficients in linear combinations

\begin{tabular}{lccc}
\hline \multirow{2}{*}{ Comfort factor } & \multicolumn{3}{c}{ component } \\
\cline { 2 - 4 } & 1 & 2 & 3 \\
\hline Auditory comfort 3 & 0.269 & 0.188 & 0.217 \\
Auditory comfort 2 & 0.268 & 0.192 & 0.224 \\
Auditory comfort 4 & 0.266 & 0.175 & 0.249 \\
Auditory comfort 1 & 0.265 & 0.198 & 0.207 \\
Auditory comfort 5 & 0.256 & 0.177 & 0.243 \\
Auditory comfort 7 & 0.240 & 0.175 & 0.245 \\
Olfactory comfort & 0.121 & 0.220 & 0.347 \\
Visual comfort 3 & 0.072 & 0.620 & 0.228 \\
Visual comfort 4 & 0.076 & 0.612 & 0.217 \\
Visual comfort 2 & 0.074 & 0.603 & 0.269 \\
Visual comfort 1 & 0.069 & 0.587 & 0.288 \\
Visual comfort 5 & 0.085 & 0.562 & 0.190 \\
Visual comfort 6 & 0.125 & 0.507 & 0.181 \\
Tactile comfort 3 & 0.094 & 0.192 & 0.758 \\
Tactile comfort 2 & 0.090 & 0.222 & 0.753 \\
Tactile comfort 1 & 0.085 & 0.265 & 0.678 \\
Tactile comfort 4 & 0.102 & 0.180 & 0.652 \\
\hline
\end{tabular}

Table 3 shows the calculated linear combination coefficient. On the basis of the linear combination coefficient, the coefficient under the comprehensive score model of the subjective evaluation index is further calculated. On the basis of the linear combination coefficient, the coefficient under the comprehensive score model of the subjective evaluation index is further calculated. The calculated results are shown in Table 4.

Table 4. Coefficient of comprehensive score model

\begin{tabular}{ll|ll}
\hline \multicolumn{4}{c}{ Coefficient of comprehensive score model } \\
\hline Auditory comfort 3 & 0.252 & Visual comfort 2 & 0.169 \\
Auditory comfort 2 & 0.253 & Visual comfort 1 & 0.164 \\
Auditory comfort 4 & 0.251 & Visual comfort 5 & 0.163 \\
Auditory comfort 1 & 0.250 & Visual comfort 6 & 0.186 \\
Auditory comfort 5 & 0.244 & Tactile comfort 3 & 0.170 \\
Auditory comfort 7 & 0.231 & Tactile comfort 2 & 0.171 \\
Olfactory comfort & 0.157 & Tactile comfort 1 & 0.166 \\
Visual comfort 3 & 0.166 & Tactile comfort 4 & 0.165 \\
Visual comfort 4 & 0.166 & & \\
\hline
\end{tabular}

Calculating the weights in final, later, all the indexes are normalized, so that their weights are integrated to 1 . The weights are arranged in ascending order according to the numerical value. What can be seen from the results in Table 5 is that the weight coefficient of olfactory comfort is the smallest, the coefficient of visual comfort and tactile comfort are very close, and auditory comfort has the largest coefficient. According to the same data 
processing method, calculating and analyzing the weight and comprehensive score coefficient respectively, from subjective evaluation data which belongs to the other eight benchmarking models, so as to lay the data foundation for the subjective and objective analysis of standard models of the same level.

Table 5. Index weight coefficient of vehicle 1

\begin{tabular}{ll|ll}
\hline \multicolumn{4}{c}{ Index weight } \\
\hline Olfactory comfort & 0.047 & Tactile comfort 2 & 0.051 \\
Visual comfort 5 & 0.049 & Visual comfort 6 & 0.056 \\
Visual comfort 1 & 0.049 & Auditory comfort 7 & 0.069 \\
Tactile comfort 4 & 0.050 & Auditory comfort 5 & 0.073 \\
Visual comfort 3 & 0.050 & Auditory comfort 1 & 0.075 \\
Tactile comfort 1 & 0.050 & Auditory comfort 4 & 0.076 \\
Visual comfort 4 & 0.050 & Auditory comfort 3 & 0.076 \\
Visual comfort 2 & 0.051 & Auditory comfort 2 & 0.076 \\
Tactile comfort 3 & 0.051 & & \\
\hline
\end{tabular}

\section{Verification and analysis of seat subjective comfort evaluation index}

Selected and compared the subjective and objective evaluation results of driver's seat of 3 standard models that in the same level, focusing on verifying whether the results of the subjective evaluation index weight coefficient of nine standard models gained by calculation is in line with the actual evaluation situation. The standard models selected in this validation analysis are medium and large SUV models of the same grade, and the subjective evaluation result is the score of consumers' evaluation of seat comfort. The objective evaluation results are the test and record results for the seats of the test models. The configurations of the three models are shown in Table 6:

Table 6. Three alternative models and configurations

\begin{tabular}{|c|c|c|c|}
\hline $\begin{array}{c}\text { Sequence } \\
\text { number }\end{array}$ & Models & Types & Seat configuration \\
\hline 1 & Ford Edge & $\begin{array}{l}\text { 2018type two-wheel } \\
\text { drive 5seats }\end{array}$ & $\begin{array}{l}\text { Electric adjustment, } \\
\text { leather fabric }\end{array}$ \\
\hline 2 & ENVISION & $\begin{array}{l}\text { 2018type } 2.0 \mathrm{~T} \text { two- } \\
\text { wheel drive }\end{array}$ & $\begin{array}{l}\text { Electric adjustment, } \\
\text { leather fabric }\end{array}$ \\
\hline 3 & HILANDER & $\begin{array}{l}\text { 2018type } 2.0 \mathrm{~T} \text { two- } \\
\text { wheel drive } 7 \text { seats }\end{array}$ & $\begin{array}{l}\text { Electric adjustment, } \\
\text { leather fabric }\end{array}$ \\
\hline
\end{tabular}

\subsection{Visual comfort evaluation and appearance dimension test}

The results of each evaluation item in consumer visual comfort were analyzed with normal distribution. The mode of scoring is calculated, that is, the comprehensive score of a certain item, as shown in Table 7. Table 8 shows the evaluation and measured data of seat size of each car model. 
Table 7. Subjective evaluation results of visual comfort

\begin{tabular}{lccc}
\hline \multirow{2}{*}{ Items } & \multicolumn{3}{c}{ Model evaluation results } \\
\cline { 2 - 4 } & Ford Edge & HILANDER & ENVISION \\
\hline Protective surface texture & 7 & 8 & 8 \\
Overall appearance & 7 & 8 & 8 \\
Cushion modelling & 7 & 8 & 8 \\
Backrest modelling & 7 & 8 & 8 \\
Headrest modelling & 7 & 8 & 8 \\
Side panel adjusting handle & 7 & 7 & 8 \\
\hline
\end{tabular}

As you can see, among three large SUV, consumers generally judged that the seat on Ford Edge is relatively poor about visual comfort, score for each project were low in comparation. For ENVISION, all the scores about subjective visual comfort items were above 8 points, and its seat is relatively popular by consumers in terms about visual comfort. Through the comparison of objective measurement, it is easily to find that all the key size of the seats on Ford Edge are basically the smallest, indicating that the overall size of the seat is relatively small. Meanwhile the seats are installed on a large SUV model. The strong contrast is also the main reason for consumers' poor evaluation to the seat's visual comfort. For seats on ENVISION, each key dimension is basically the median of all the three models', getting the best comprehensive evaluation of consumers' visual comfort. This indicating that, for large SUV, consumers have the best visual comfort experience for seats with moderate appearance size.

The seat appearance size which over small or over big, often make consumer visual feel uncomfortable.

Table 8. Measurement of seat appearance dimensions

\begin{tabular}{lccc}
\hline \multirow{2}{*}{\multicolumn{1}{c}{ Items }} & \multicolumn{3}{c}{ Measurement results of appearance dimensions } \\
\cline { 2 - 4 } & Ford Edge & HILANDER & ENVISION \\
\hline Cushion length/mm & 349.9 & 380.0 & 352.1 \\
Cushion overall width /mm & 483.2 & 504.1 & 494.1 \\
Seat flank height /mm & 51.4 & 38.2 & 38.1 \\
Cushion thickness /mm & 67.8 & 77.0 & 87.2 \\
Cushion angle / & 14.3 & 20.9 & 15.5 \\
Backrest length /mm & 655.6 & 678.2 & 607.7 \\
Backrest width-R points /mm & 424.4 & 531.3 & 501.4 \\
Cushion thickness /mm & 120.5 & 121.2 & 126.9 \\
Backrest flank height /mm & 78.3 & 74.4 & 80.4 \\
Seat height/mm & 950.3 & 1035.4 & 1030.7 \\
Backrest and cushion angle $/{ }^{\circ}$ & 98.9 & 99.0 & 97.8 \\
\hline
\end{tabular}

\subsection{Auditory comfort evaluation and vibration and noise test}

Using normal distribution analysis to process the results of each evaluation item in the consumer's auditory comfort, calculating the mode of scoring, that is the comprehensive score of a certain item, as the ones shown in Table 9. 
Table 9. Subjective evaluation results of auditory comfort

\begin{tabular}{lccc}
\hline \multirow{2}{*}{\multicolumn{1}{c}{ Items }} & \multicolumn{3}{c}{ Model evaluation results } \\
\cline { 2 - 4 } & Ford Edge & HILANDER & ENVISION \\
\hline Seat forward-backward adjustment & 8 & 8 & 8 \\
Backrest angle adjustment & 8 & 8 & 8 \\
Seat angle adjustment & 8 & 8 & 8 \\
Seat height adjustment & 8 & 8 & 8 \\
Headrest adjustment & 8 & 8 & 8 \\
Waist support up and down-back & 8 & 8 & 8 \\
and forth adjustment & 8 & 8 & 8 \\
Armrest adjustment & & 8 & 8 \\
\hline
\end{tabular}

The measurement results and comprehensive evaluation results value of each key part of the seat are shown in Table 10.

Table 10. Measurement results of vibration and noise of seats

\begin{tabular}{lccc}
\hline \multirow{2}{*}{\multicolumn{1}{c}{ Measuring point location }} & \multicolumn{3}{c}{ Noise value /dB } \\
\cline { 2 - 4 } & Ford Edge & HILANDER & ENVISION \\
\hline Front of headrest & 53.4 & 52.6 & 56.7 \\
Front of backrest & 51.2 & 54.6 & 54.4 \\
Front of seat slideway & 47.4 & 57.5 & 47.4 \\
Backrest adjuster (external) & 48.2 & 58.9 & 48.3 \\
Seat slideway(external) & 48.7 & 57.6 & 48.5 \\
Backrest adjuster(internal) & 51.4 & 58.4 & 48.7 \\
Seat slideway(internal) & 49.6 & 58.7 & 48.6 \\
Back of headrest & 51.7 & 51.8 & 55.1 \\
Back of backrest & 52.5 & 52.3 & 51.7 \\
Comprehensive noise value & 50.5 & 55.8 & 51.1 \\
\hline
\end{tabular}

It is clear that the subjective evaluation results of consumers' auditory comfort of the three alternative models are completely consistent, both of them are excellent evaluation results. It indicates that, when consumers adjusted the seats of several alternative models directly, there was no obvious abnormal sound and noise, to make consumers have poor auditory comfort. But after further analyzing the test results of objective test, certain differences still can be found on three models, under the same test conditions or road conditions. Finally, we found that the Ford Edge seats had the lowest comprehensive noise, $50.5 \mathrm{~dB}$, and Highlander seats had the highest one, 55.8dB. Therefore, Ford Edge seats received more excellent objective evaluation result of the auditory comfort.

\subsection{Olfactory comfort evaluation and odor evaluation}

Consumers' of subjective evaluation comprehensive score of olfactory comfort of the vehicle is shown in Table 11. The seat objective odor evaluation was judged by professional engineers, and the scores were shown in Table 11. From the results comparison we can see that consumers have the same sense of olfactory comfort for the three alternative models, the objective evaluations from professional engineers performed differences. The Highlander got a worse score than another two models. This also makes sure that we can evaluate seat comfort more accurately from different dimensions. 
Table 11. Subjective and objective evaluation results of olfactory comfort

\begin{tabular}{lccc}
\hline \multirow{2}{*}{ Items } & \multicolumn{3}{c}{ Model evaluation results } \\
\cline { 2 - 4 } & Ford Edge & HILANDER & ENVISION \\
\hline Seat odor (subjective) & 8 & 8 & 8 \\
Odor evaluation & 3.5 & 4.0 & 3.5 \\
Odor type & Foaming odor, Leather irritation odor, Stink, Plywood stink \\
\hline
\end{tabular}

\subsection{Tactile comfort evaluation and chair foam hardness test}

Consumers' of subjective evaluation comprehensive score of tactile comfort of the vehicle is shown in Table 12:

Table 12. Subjective evaluation results of tactile comfort

\begin{tabular}{lccc}
\hline \multirow{2}{*}{\multicolumn{1}{c}{ Items }} & \multicolumn{3}{c}{ Model evaluation results } \\
\cline { 2 - 4 } & Ford Edge & HILANDER & ENVISION \\
\hline The touch of the protective surface & 8 & 8 & 7 \\
Hand pressure to cushion & 7 & 8 & 7 \\
Hand pressure to backrest & 8 & 8 & 7 \\
Hand pressure to headrest & 7 & 8 & 7 \\
\hline
\end{tabular}

Testing the hardness of seat cushion and backrest, and the test results were shown in Table 13.

Table 13. Measurement of seat hardness value

\begin{tabular}{cccc}
\hline \multirow{2}{*}{ Items } & \multicolumn{3}{c}{ Hardness value /N } \\
\cline { 2 - 4 } & Ford Edge & HILANDER & ENVISION \\
\hline Cushion hardness value & 27.7 & 18.3 & 22.6 \\
Backrest hardness value & 14.1 & 12.4 & 8.3 \\
\hline
\end{tabular}

It is obviously that consumers made the highest tactile comfort evaluation for the seats on Highlander. By comparing the objective data, the Highlander seat cushion got the minimum hardness value among the three models, and the hardness of the backrest is the middle value of the three models. That is to say, for the tactile comfort of the seat cushion, consumers prefer soft foaming materials, so that buttocks can have better comfort after riding the car. For the tactile comfort of the backrest, consumers prefer the materials which have moderate hardness, so that consumers' back can be supported better during the driving process, meanwhile, the backrest can also maintain comfort.

\subsection{Comprehensive evaluation score and objective test results of seat perceived comfort}

According to the research results on the perception factor weight coefficient in Chapter 2 . Calculating comprehensive score of subjective evaluation for each car. As Table 14 showing, Highlander has the highest subjective score of 7.956 , followed by ENVISION with 7.77, Ford Edge with 7.665, locate at the last one.

Table 14. Calculation of subjective evaluation comprehensive scores

\begin{tabular}{lccc}
\hline \multirow{2}{*}{\multicolumn{1}{c}{ Items }} & \multicolumn{3}{c}{ Model evaluation results } \\
\cline { 2 - 4 } & Ford Edge & HILANDER ENVISION \\
\hline Auditory comfort factor & 8 & 8 & 8 \\
Visual comfort factor & 7 & 7.8 & 8 \\
Olfactory comfort factor & 8 & 8 & 8 \\
Tactile comfort factor & 7.5 & 8 & 7 \\
\hline Subjective evaluation comprehensive score & 7.665 & 7.956 & 7.77 \\
\hline
\end{tabular}


Next, we compare and analyze the subjective evaluation scores with the objective test results of each item. First of all, we analyze the appearance size data, and find that about $60 \%$ of the appearance size data presents a positive correlation with the subjective evaluation comprehensive score. In other words, within a certain range, the larger the appearance size means the higher subjective evaluation score from consumers. These dimensions include the length of the seat, the overall width of seat, the height of seat flank, the angle of cushion, the backrest width-R point, the seat height, etc., as shown in Table 15.

Table 15. Comparison of overall score of appearance size data and subjective evaluation comprehensive score

\begin{tabular}{lccc}
\hline \multirow{2}{*}{ Items } & \multicolumn{3}{c}{ Evaluation results } \\
\cline { 2 - 4 } & Ford Edge & HILANDER & ENVISION \\
\hline Cushion length /mm & 349.9 & 380.0 & 352.1 \\
Cushion overall width /mm & 483.2 & 504.1 & 494.1 \\
Seat flank height /mm & 51.4 & 38.2 & 38.1 \\
Cushion angle / & 14.3 & 20.9 & 15.5 \\
Backrest width-R points /mm & 424.4 & 531.3 & 501.4 \\
Seat height /mm & 950.3 & 1035.4 & 1030.7 \\
\hline Subjective evaluation comprehensive score & 7.665 & 7.956 & 7.77 \\
\hline \multicolumn{1}{c}{ Later, we analyze the vibration noise test results, through the Table 16, you can see }
\end{tabular}

the comprehensive noise value test results for all the selected standard models, HILANDER $(55.8 \mathrm{~dB})>$ ENVISION $(51.1 \mathrm{~dB})>$ Ford Edge $(50.5 \mathrm{~dB})$, and the relationship of subjective evaluation comprehensive score between three models is HILANDER $>$ ENVISION $>$ Ford Edge. This result is contrary to our common perception that the higher the seat noise value is, the worse the consumer comfort is and the lower the subjective evaluation score is. In other words, within a certain range for noise value (when the noise value is small), the higher noise value of seat assembly is, the higher subjective evaluation score of perceived comfort is, and the better comfort experience of consumers is.

Table 16. Comparison between the measurement results of vibration and noise of seats and the subjective evaluation comprehensive scores

\begin{tabular}{cccc}
\hline \multirow{2}{*}{ Measuring point location } & \multicolumn{3}{c}{ Noise value /dB } \\
\cline { 2 - 4 } & Ford Edge & HILANDER & ENVISION \\
\hline Front of headrest & 53.4 & 52.6 & 56.7 \\
Front of backrest & 51.2 & 54.6 & 54.4 \\
Front of seat slideway & 47.4 & 57.5 & 47.4 \\
Backrest adjuster (external) & 48.2 & 58.9 & 48.3 \\
Seat slideway(external) & 48.7 & 57.6 & 48.5 \\
Backrest adjuster(internal) & 51.4 & 58.4 & 48.7 \\
Seat slideway(internal) & 49.6 & 58.7 & 48.6 \\
Back of headrest & 51.7 & 51.8 & 55.1 \\
Back of backrest & 52.5 & 52.3 & 51.7 \\
\hline Comprehensive noise value & 50.5 & 55.8 & 51.1 \\
\hline Subjective evaluation & 7.665 & 7.956 & 7.77 \\
comprehensive score & &
\end{tabular}

For the odor evaluation results, there is no obvious correlation between evaluation results and consumers' subjective evaluation scores. This is mainly due to the fact that the scores of odor evaluation are relatively simple, the standard models are mostly the ones that 
more comfortable to consumers, and also due to the consumers have a low sensitivity to odor.

Table 17. Comparison of odor evaluation results and subjective evaluation comprehensive scores

\begin{tabular}{lccc}
\hline \multirow{2}{*}{ Items } & \multicolumn{3}{c}{ Evaluation results } \\
\cline { 2 - 4 } & Ford Edge & HILANDER & ENVISION \\
\hline $\begin{array}{l}\text { Subjective evaluation } \\
\text { comprehensive score }\end{array}$ & 7.665 & 7.956 & 7.77 \\
\hline Odor evaluation & 3.5 & 4.0 & 3.5 \\
\hline Odor type & Foaming odor, Leather irritation odor, Stink, Plywood stink
\end{tabular}

Odor type $\quad$ Foaming odor, Leather irritation odor, Stink, Plywood stink

The test of seat hardness value is shown in Table 18 .

Table 18. Comparison of chair hardness value measurement subjective evaluation and comprehensive scores

\begin{tabular}{lccc}
\hline \multirow{2}{*}{ Items } & \multicolumn{3}{c}{ Hardness value /N } \\
\cline { 2 - 4 } & Ford Edge & HILANDER & ENVISION \\
\hline Cushion hardness value & 27.7 & 18.3 & 22.6 \\
Backrest hardness value & 14.1 & 12.4 & 8.3 \\
\hline Subjective evaluation comprehensive score & 7.665 & 7.956 & 7.77 \\
\hline
\end{tabular}

As for the hardness value of the seat cushion, we find that, within a certain range, higher subjective evaluation comprehensive score given by consumers means the seat cushion has lower hardness value, and also means well comfort. It indicates that consumers tend to choose the seat and cushion that with lower driving hardness and softer foam. To the hardness value of the backrest, we find that when the hardness value is moderate, consumers' subjective evaluation comprehensive score is the highest. When the hardness value increases or decreases, the consumers' comfort score will decrease. We can see that the backrest hardness value has higher sensitivity to consumers' comfort evaluation. This is directly related to the fact that consumers are more prone to tired at the back part during riding.

\section{Conclusion and Prospect}

Through calculating the score of subjective comfort factor on three standard model which in the same level, also horizontal contrasting the calculated results and the corresponding objective test results, we can see there is no obvious correlation between the odor evaluation and the subjective evaluation score, while the noise results showed a negative correlation with the subjective evaluation score, which was not consistent with our expectation of subjective comfort evaluation.

The objective measurement results of seat size are positively correlated with the subjective evaluation scores within a certain range. Contrasting and analyzing test value of seat hardness results and subjective evaluation, what can be found is that consumers are more inclined to choose soft cushions. As for choosing backrest, its subjective evaluation score is not high if its hardness is too high or too low. It also proves that the consumers preferred those backrests with moderate hardness. So, the subjective scores of these two parts are in line with the expected effect.

Subjective comfort score does can reflect the seat comfort degree in real. However, due to the low sensitivity of consumers to odors and sounds, or the sample data of standard models in the same level is incomplete, these reasons result to the discrepancy to smell and 
noise evaluation and expectations. In the later stage, deeper research will be launched to complete the calculation of subjective evaluation index model.

\section{References}

1. Rude. Wang. BQResearch on the evaluation of BQ company's automobile perceived quality [D]. Beijing University of Technology (2016)

2. Yunchao. Dong. SPSS software was used to analyze the correlation between nominal variables $[\mathrm{J}]$. School of statistics and information, Shanghai University of international business and Economics (2019)

3. JingZhang. Research on Influencing Factors of executive ability of salesmen in Shanghai Volkswagen 4S store based on factor analysis [D]. Chang'an University, (2016)

4. Kun-Hua X U, Huang J W, Wang D M, et al. Comprehensive evaluation of factors influencing sultone in sodium $\alpha$-olefin sulfonate based on SPSS factor analysis [J]. China Surfactant Detergent \& Cosmetics (2019)

5. Li Q, Lei J, Tian W, et al. Risk estimation of large complex bridge construction based on factor analysis [J]. IOP Conference Series: Materials Science and Engineering, (2020) 